\title{
Interactive comment on "Modeling the annual cycle of daily Antarctic sea ice extent" by Mark S. Handcock and Marilyn N. Raphael
}

Anonymous Referee \#2

Received and published: 29 January 2020

\section{General Comments}

The paper proposes a statistically based framework for investigating the annual cycle in Antarctic sea ice extent (SIE). The paper delves into the different drivers of variation in the annual cycle, with a focus on the amplitude and the phase. Many researchers (myself included) who are interested in Antarctic Sea Ice and its changes over the satellite era, have or are, pondering over the drivers of change in SIE. I would recommend that the paper is published with minor revisions, much of which from my personal perspective, focus on the accessibility of the paper.

This work will be of great interest to the entire community interested in Antarctic sea 
ice, from researchers focused on SIE through to biologists, glaciologists and those involved in the operational aspects of Antarctic science. To that end, I would ask the authors to consider whether the paper can be improved in terms of its accessibility. Reviewing this, I am required to read every line, and as such, I found that I needed several "sittings" to complete my first read through. I would deal with a section, but I would then need a break of several hours before I felt ready to tackle the next section. The nature of this work, the detail and effort that has gone into developing the method, does to an extent require this level of complexity, but my fear is that it might push other readers away, meaning they miss the crucial detail within the methods. To this end, I have a few suggestions for improving the accessibility:

\section{A) Introduction}

Lines 23-37 quickly bounce from introducing the annual cycle and its year on year variability to delving into issues surrounding satellite retrieval then into complexities regarding the duration of the record itself. I think this should be split up slightly, particularly the focus on the components of the annual cycle in Lines 23-26. Amplitude and phase are crucial throughout much of the paper and I think it would be of great value for the authors to spend some time here, defining them and why they are important. I would also remove the brief mention of the regional variations in the annual cycle, the body of this work will focus on pan-Antarctic SIE, and while there are interesting developments to this work looking at specific regions, it is fairly, not covered in the majority of this paper.

Once the components of the annual cycle are defined, it will be then easier to outline why they are affected by the current retrieval methods and the duration of the record. This then allows a better set up for why this work is necessary.

I would delete Lines 38-41 (up to and including "climate data"). No specific examples of other annual climate cycle issues are outlined, nor does it seem that the methods used here are applied elsewhere (if they are, then please state this with the example

Printer-friendly version

Discussion paper 
more clearly). Removing these lines would allow better flow from outlining the issues into your over-arching aims.

Line 51 is a good example of the general accessibility of the text; "the mathematical and stochastic representation of proximate forces" is potentially obtuse. The (very) similar sentence that follows from Line $52-53$ is far more accessible to a less statistically minded reader.

B) Methodology and Results

Each process is defined with respect to the model, previous models and the statistical analysis involved. What I think would benefit this section is for an introductory section at the beginning of Section 3 that defines each term for: âĂć Annual Cycle âĂć Invariant Annual Cycle âĂć Amplitude and Phase Adjusted Annual Cycle

Highlighting their importance to understanding the cycles and the changes. The final line for each of these would point to the following section where they are defined and their results discussed. By creating this section, a reader can easily refresh themselves as to what is each of these components, as that is crucial to understanding the results.

The section would also benefit from the results for each cycle being a new paragraph to ensure that they stand out, currently in most of the sections it runs straight from the methodology behind the cycle into its result. This runs the risk of the result being missed by readers.

\section{Main Comments - Figures}

\section{Figure 1}

The smooth annual cycle as a blue line is not distinguishable as blue. I would change the colour so that it can be resolved by the reader, even if that was black, which is what the line mostly appears to me currently (both on screen and in print). Figure 1 also sets a convention that Day 0 is the start of the cycle and Day 365 is the end of the cycle. However, the annual cycle is rarely exactly 365 days, it is "on average" but year on year

Printer-friendly version

Discussion paper 
it is not. How does this impact on both the figures and also the paper analysis? This should be addressed in the body text of the paper.

Figure 2

Please label the panels as A and B. In general both panels are too small to properly resolve the detail within the image, particularly from the lines that overlap. In $A$, the green and black are nearly indistinguishable to my eye.

Figure 3

The title over the figure appears to be incomplete. Given a title doesn't appear on the other figures is this an error for it to still remain?

Figure 5 and Figure 6

I'm not a fan of green, blue and black lines on the same figure, they are very hard to resolve, particularly the green and black which heavily overlap. The same issue also applied to Figure 6.

\section{Figure 7}

In a similar vein to Figures $5 \& 6$, the use of maroon and red on the same figure makes the figure harder to interpret. The figure legend also needs to be significantly bigger to make it more readable.

\section{Specific (Line-by-Line) Comments}

Line 16-17: The ordering of the references is ad-hoc, neither in publication date order or alphabetical order based on first author initial. In Line 20, they are ordered by date of publication, please adjust Line 16 to match.

Line 23: In the abstract (Line 2) you state that the SIE minima is in March; here in Line 23 you state it is late February. Please ensure consistency between these two dates. (See also Line 230-231 comments and Figure 1 comments) 
Line 24: I would suggest altering this line to read: "The growth from minimum (trough) to maximum (peak) is slower than the retreat from maximum to minimum". Your use of trough refers to the minima, not the slope getting there, so I think defining both terms straight away fits better.

Line 39 (and others): Some sections of the paper, such as here use an example reference (Stine et al., 2009) is followed by "e.g.,". Elsewhere in the paper it precedes the reference (i.e. Line 29 "(e.g. Comiso and Steffen, 2001)"). I would prefer the Line 29 example, but either way is acceptable as long as it is consistent throughout the paper.

Lines 58-59: The outline of the sections is not consistent with the body text. Results are in Section 3 alongside the Methods and Conclusions are in Section 4.

Line 81: $T=2019$. I was unsure if this should be $T=2019.000 ; T=2019.999$ or something in between. Could this be clarified.

Line 89: I would appreciate a short (1 or 2 lines) explaining why you chose not to consider this data further. To raise it as a possible way to increase your temporal range and then simply dismiss it feels incomplete to me.

Line 230-231: Here the minima is listed a mid-February, which is different to the use of March (abstract) and late-February (i.e. Line 23). The consistency of the definition of the minima throughout the paper would be useful (albeit tricky due to the variability in the occurrence of the minima; as mentioned in the comments on Figure 1, this could do with being addressed earlier in the paper)

Lines 236-239: Ice budget analysis work (i.e. Holland \& Kwok, 2012; Holland, 2014; Pope et al., 2017; Holmes et al., 2019) has indicated that the surface winds play a role (advection and divergence terms within the budget) throughout the whole of West Antarctica and into the Weddell Sea in both the advance and the retreat of sea ice. I would mention this work here with respect to the drivers of the advance and retreat of sea ice in addition to the modelling study mentioned. 
Line 239-240: the sudden use of months (when most of the time dimension to date in the paper has been in days) is unnecessary and makes for awkward flow. I would remove 7.5 months and 4.5 months from these lines, as it would clean up the sentence which has to many "-" in it, making for a poor flow.

Line 349: The authors should be praised for being so diligent in making their code accessible and open to peer review.

Interactive comment on The Cryosphere Discuss., https://doi.org/10.5194/tc-2019-203, 2019. 